\title{
Prospective community study of family stress and anxiety in (pre)adolescents: the TRAILS study
}

\author{
Floor V. A. van Oort · Frank C. Verhulst • \\ Johan Ormel · Anja C. Huizink
}

Received: 25 February 2009/Accepted: 9 September 2009/Published online: 13 October 2009

(C) The Author(s) 2009. This article is published with open access at Springerlink.com

\begin{abstract}
For prevention of anxiety in children and adolescents, it is important to know whether family stress is a predictor of anxiety. We studied this in 1,875 adolescents from the Tracking Adolescents' Individual Lives Survey (TRAILS) who were followed up for 2 years, from age 1012 to 12-14 years. Adolescents reported anxiety and depression symptoms at both assessments, and parents reported family stress (family dysfunction and parenting stress) at the first assessment. Family dysfunction was not associated with future anxiety, whereas high parenting stress was. Furthermore, family dysfunction was more strongly associated with anxiety than with depression, whereas parenting stress was more strongly associated with depression. Level of parental psychopathology explained part of the association of family stress with anxiety. The associations were modest and the understanding of the origins of adolescents' anxiety will require identifying other factors than family stress that account for more of the variance.
\end{abstract}

Keywords Anxiety · Family functioning . Parenting stress $\cdot$ Prospective study $\cdot$ Adolescents

F. V. A. van Oort $(\varangle)$. F. C. Verhulst · A. C. Huizink Department of Child and Adolescent Psychiatry, Erasmus MC, P.O. Box 2060, 3000 CB Rotterdam, The Netherlands e-mail: f.vanoort@erasmusmc.nl

J. Ormel · A. C. Huizink

Interdisciplinary Center for Psychiatric Epidemiology, University Medical Center Groningen, P.O. Box 30.001, 9700 RB Groningen, The Netherlands

\section{Introduction}

Research has established a strong link between environmental stress and internalising problems in children [17]. For children their family is their closest environment. Important positive aspects of the family environment include supportive and facilitative interactions, and the absence of conflict. Not surprisingly, studies have shown that family stress is associated with anxiety and depression. However, most of these studies compared children who already had anxiety symptoms or an anxiety disorder with non-anxious children (e.g. [8, 19, 26, 37]), or children from anxious parents with those from non-anxious parents [5, 23]. Furthermore, these studies have been diverse with respect to definition and measurement of family stress (e.g. family assessment questionnaires, clinician-rated measures, and observational tasks), making comparisons across studies difficult. The different methods do not measure the same concepts: observational tasks are mostly in laboratory setting, from which we do not know whether they generalize to naturalistic daily family functioning, whereas questionnaires measure daily family functioning, but are subject to the situational interpretation of the informant. Longitudinal studies on the predictive effect of family stress on child anxiety are scarce [5, 31], making it very difficult to give an interpretation of the direction of the association. It is very well possible that anxious children provoke stress in families, but also that children become anxious as a result of their exposure to family stress. Probably, both influences will be present, although, one may be stronger than the other. We will focus on family stress as a predictor of anxiety in children.

In studies of the role of family stress in anxiety, it is important not only to look at stress within the parent-child dyad, but to include overall family dysfunction, which is 
not restricted to the parent-child dyad [12, 14]. Family stress has been found to adversely affect the quality of care giving, and parent-child interactions. Adverse effects on care giving quality include insecure attachment [25], maternal unresponsiveness, and use of negative parenting styles, such as less affection, supervision, and autonomy $[32,36,37]$. Families of anxious children were found to be more controlling, more rejecting, and less intimate [29, 34]. These consequences of family stress may in turn contribute to the child's perception and appraisal of life events and ways of coping with stressful situations, and eventually lead to anxious behaviour [28, 45]. Family stress may be the result of poor parental mental health and family composition, and so form an important mediator between parental and child mental health problems. For example, in larger families there might be a higher chance for a chaotic and conflictual life, or in other words more family stress. Single-parent status may be a precursor of family stress as discord and conflict in families often occurs before, during and after a divorce [5, 8, 12, 40]. Other studies have already demonstrated the link between parental mental health and family stress $[8,12,40]$.

Few studies have explored whether family or parenting variables are specific to anxiety versus other internalising disorders [5, 45]. Furthermore, for prevention of anxiety in children and adolescents, it is important to know whether family stress is a predictor of childhood anxiety. However, most research has been cross-sectional in design, and there is lack of studies demonstrating the role of family stress in the development of anxiety. We studied the role of family stress in a large community sample of preadolescents and adolescents followed up for 2 years.

This paper addresses the following questions: (1) Is family stress related to current anxiety symptoms? (2) Does family stress predict anxiety symptoms 2 years later? (3) Are these associations independent of comorbid depression? and (4) Is the association of family stress with anxiety a result of parental mental health or unfavourable family composition?

\section{Methods}

\section{Design}

The TRacking Adolescents' Individual Lives Survey (TRAILS) is a prospective cohort study of Dutch early adolescents aged 10-12 years, who are followed biennially. The present study used data from the first (20012002) and second (2003-2004) assessment wave. The TRAILS target sample consisted of young (pre)adolescents from five municipalities in the North of the Netherlands, including both urban and rural areas. These municipalities were requested to give names and addresses of all inhabitants born between 10-01-1989 and 30-09-1990 (first two municipalities) or between 10-01-1990 and 30-09-1991 (last three municipalities), which yielded 3,483 names. Subsequently, primary schools within these municipalities were requested to participate. Young adolescents and their parents were approached with the question to participate in TRAILS, only if their schools had agreed to take part in TRAILS. This means that of the 135 primary schools within the municipalities, $122(90.4 \%)$ schools participated, accommodating $90.3 \%$ of the listed young adolescents. More details about the sample selection have been published elsewhere [11].

\section{Population}

Of all subjects who were approached at wave one $(N=3,145), 6.7 \%$ were excluded. The exclusion criteria were (1) adolescents incapable to participate because of severe intellectual disability or a serious physical illness or handicap and (2) Dutch-speaking parent or parent surrogate not available, and not feasible to administer the measurements in parent's own language. Of the remaining 2,935 young adolescents, $76 \%$ participated in the study at wave one $(N=2,230$, mean age 11.1 years, SD 0.55 , with $51 \%$ girls). To enable a comprehensive analysis of non-response bias, information on mental health determinants and outcomes was obtained from the teachers of responders and non-responders. Responders and non-responders did not differ with respect to family composition or teacher-rated problem behaviour. No differences were found between responders and non-responders regarding associations between socio-demographic variables and mental health outcomes. Yet, responders had a lower proportion of loweducated parents than non-responders (32.6 vs. $44.2 \%$ ) [11].

At the second wave, 2-3 years after the first wave, 2,149 adolescents participated ( $96 \%$ of wave one participants), of whom 2,089 had complete data for anxiety at both waves ( $94 \%$ of wave one participants). For the analyses we used only adolescents with complete data for all of the measures $(N=1,875)$. Respondents who were excluded because of missing values on one or more of the variables of interest, did not differ from those included in anxiety scores at both waves, in family dysfunction, parenting stress, sex, age, and parental internalising problems. Those excluded from analyses more often lived with one parent at wave one, had lower parental socio-economic position, and a higher score for parental externalising problems.

\section{Measures}

In order to reduce the possibility that the relationships found in any analysis were because of the use of the same 
informant, we assessed anxiety from the perspective of adolescents, and family stress from the perspective of the parents. Anxiety and depression were measured at both assessments, whereas family stress, parental mental health, family composition, and socio-economic position were measured at the first assessment only.

\section{Anxiety}

The Revised Child Anxiety and Depression Scale (RCADS) [7] was used to assess anxiety symptoms. The RCADS is a revision of the Spence Children's Anxiety Scale (SCAS) [39]. Results of the study of Chorpita et al. [7] showed that the RCADS has sufficient reliability and a strong concurrent and discriminant validity with the Revised Children's Manifest Anxiety Scale (RCMAS) and the Children's Depression Inventory (CDI). Both the RCMAS and CDI correspond to earlier editions of DSM anxiety and depressive disorders $[7,16]$. The RCADS is a self-report questionnaire with 47 items that are scored on a 4-point scale $(0=$ never, $1=$ sometimes, $2=$ often, 3 = always). The questionnaire covers symptoms corresponding to DSM-IV dimensions of anxiety disorders and depressive disorder: generalised anxiety disorder, separation anxiety disorder, social phobia, panic disorder, obsessive-compulsive disorder, and major depressive disorder. The factor structure for all six scales together was confirmed by confirmatory factor analysis in the TRAILSsample at wave one (fit indices of NNFI $=0.96$, RMSEA $=0.05$, and SRMR $=0.05$, indicating adequate fit to the sample data) [16]. We used the total score of anxiety symptoms, as we did not have hypotheses for specificity according to subtype of anxiety. The internal consistencies were (respectively wave one/wave two) $0.91 /$ 0.93 for anxiety and $0.71 / 0.81$ for depression. The anxiety and depression scale scores were calculated as mean item scores.

\section{Family stress}

To measure family dysfunction we used the subscale General Functioning of the Family Assessment Device (FAD) [15]. The FAD is a 60-item self-report measure of family functioning. Respondents have to report their agreement with statements about families on a 4-point scale. The FAD describes emotional relationships and functioning within the family. The 12-item General Functioning scale can be used as a short version of the FAD. Family dysfunction measured with this scale, describes avoiding discussing concerns or fears, having bad feelings within the family, not being able to turn to each other for support or to confide in each other, not being able to talk about sadness or express feelings to each other, difficulty in making decisions, not accepting family members as they are, and difficulty planning family activities. Previous studies have shown adequate test-retest reliability and low correlations with social desirability [30]. The general functioning scale has been shown to be a valid and reliable measure of family functioning in surveys [6]. The internal consistency of the scale in our sample was high: 0.85 . We divided our sample into two groups: one with good family functioning (lowest three quartiles), and one with dysfunction (upper quartile, cutoff level: 2.00 [30]).

Parenting stress was measured with the Dutch shortversion (25 items) of the Parental Stress Index (PSI) [1]. This short version of the PSI has shown excellent internal consistency (Cronbach's $\alpha=0.92-0.95$ ), and differentiates between clinical and non-clinical groups [10]. It contains items in the parent and child domain. We used the items in the parent domain to minimize overlap with the anxiety and depression items of the RCADS. The parent domain consists of items from the original PSI factors "Sense of competence" (6 items), "Attachment" (1 item), "Depression" (3 items) and "Parental Health" (1 item). A typical item is "I feel trapped by my responsibilities as a parent". The internal consistency of the parent scale was 0.86. As with family dysfunction, we divided our sample into two groups: one with medium/low parenting stress (lowest three quartiles) and one with high parenting stress (upper quartile, cutoff level 1.12). The decision to dichotomize the PSI and FAD was based on the finding of Bogels et al. [5, 23], that there is more evidence for extremes in family stress to be associated with child anxiety than for continuous measures of family stress.

\section{Parental mental health and family composition}

Lifetime parental mental health with respect to depression, anxiety, substance dependence and antisocial behaviour was measured with the TRAILS Family History Interview (FHI) at the first wave [33]. A description of DSM-IV symptoms characterising each of the mental health dimensions was presented to the parental informant, typically the mother (94\%), through a vignette. The parent was then asked about lifetime occurrence, professional treatment, and medication use. Based on the interview, for each dimension, parents could be allocated to one of three categories: 0 , probably never had an episode; 1 , probably yes; 2 , probably yes with treatment and/or medication (or police contact in the case of antisocial behaviour). Prevalence rates for parental depression, anxiety disorders, substance dependence, and antisocial behaviour were comparable to CIDI-DSM-IV lifetime rates (with the exception of paternal anxiety and substance use abuse which were lower) [4]. Indices of parental internalising and externalising disorders (based on the number of lifetime disorders) were calculated separately. 
The construction of these familial vulnerability indices was based on a twin modelling study investigating genetic risk factors for common psychiatric and substance use disorders conducted by Kendler et al. [20, 43]. They found evidence for two genetic common factors: one externalising (with high loadings on adult antisocial behaviour, conduct disorder, alcohol dependence, and other drug abuse or dependence) and one internalising (with high loadings on, major depression, generalised anxiety disorder and phobia). Paternal and maternal indices were combined. Internalising disorders comprised of anxiety and depression; externalising disorders comprised of substance dependence and antisocial behaviour. To calculate the vulnerability indices we summed the presence of lifetime disorders and multiplied these rates with weights [20,43]: internalising disorder $=$ $0.54 \times$ (depression mother + depression father $)+0.43 \times$ (anxiety mother + anxiety father), and externalising disorder $=0.61 \times($ substance dependence mother + substance dependence father $)+0.47 \times$ (antisocial behaviour mother + antisocial behaviour father).

Parental current internalising problems were assessed with the Depression Anxiety Stress Scales 21 (DASS-21), which is a short form of the 42-item self-report measure of depression, anxiety, and stress (DASS) [24]. The DASS-21 consists of three self-report scales with seven items each. Items were rated on a 4-point severity scale, and measured experiences over the past week. Data were available for only one parent, typically the mother $(94 \%)$, who filled out the questionnaire. Internal consistencies of the three scales were 0.83 for depression, 0.78 for anxiety, and 0.86 for stress. In a large population sample of adults in the United Kingdom the DASS-21 showed high reliability and the factor structure of the DASS was successfully replicated [18].

Two aspects of family composition were used: number of children in the family (one, two, three, four or more), and number of parents in the family (one vs. two).

\section{Socio-economic position}

Socio-economic position was measured with five indicators: educational level (father/mother, five levels ranging from 'Elementary education' to 'University'), occupation (father/mother, nine levels of the International Standard Classification of Occupations ranging from 'Elementary Occupations' to 'Legislators, Senior Officials and Managers'), and family income ( $<€ 600 /$ month to $>€ 3,500 /$ month in 9 steps). Each of the variables was standardized (zscores), and the mean of the five standardized variables was used as score of socio-economic position [20, 43]. The internal consistency was satisfactory (Cronbach's alpha 0.84 ), indicating that these variables can be considered to represent the socio-economic position of the family. The lowest $25 \%$ of scores were considered to be low socio-economic position, the highest $25 \%$ to be high socioeconomic position, and the rest were labelled middle socioeconomic position. Socio-economic position was lower in the family dysfunction group than in the group with good family functioning (Chi-square 41.80, $2 d f, P<0.0001$ ); therefore, we adjusted all our analyses for socio-economic position.

Analysis

Statistical analysis was carried out using SAS software (SAS Release 9.1, SAS institute Inc., Cary, NC, USA). We used random coefficient analysis (RCA) to model the relationship of family variables (wave one), with adolescent's anxiety at both wave one (10-12 years) and wave two (12-14 years) simultaneously [42]. RCA takes into account the correlation between anxiety scores at age 1012 and age 12-14 years. This approach provides a valid and robust estimation of variance, even when there is a known correlation between multiple outcome measures within subjects. The coefficient of the interaction of family variables with time is equivalent to that produced in a standard regression model, in which change in anxiety score is the response variable and the family variable is entered as the predictor variable. The RCA approach, however, provides information on the relationship of risk factors (here, family dysfunction and parenting stress) with outcomes, at each age, which is not provided in a standard regression analysis of change. The basic model is displayed in the following equation: $Y=\alpha+\beta_{1}$ (time) $+\beta_{2}$ (family functioning $)+\beta_{3}$ (parenting stress) $+\beta_{4}$ (family dysfunction $\times$ time $)+\beta_{5}$ (parenting stress $\times$ time $)$, where adolescent's outcome $(Y)$ is anxiety measured by the RCADS; time $=1$ at wave two (i.e. age $12-14$ ) and 0 at wave one (i.e. age 10-12); family dysfunction $=1$ if score on the FAD is in the upper quartile and 0 if score on the FAD is below the upper quartile; parenting stress $=1$ if score on the PSI is in the upper quartile (high parenting stress) and 0 if score on the PSI is below the upper quartile. Coefficient $\beta_{2}$ represents the difference between family dysfunction and medium/high family functioning in anxiety score at age 10-12, and coefficient $\beta_{3}$ the difference in anxiety between high versus medium/low parenting stress at age 10-12. All analyses were adjusted for sex and socio-economic position, which are not displayed in the equation above. We present the difference in anxiety score between dysfunction and medium/high family functioning at age 10-12 $\left(\beta_{2}\right)$ and at age 12-14 $\left(\beta_{2}+\beta_{4}\right)$ and the level of significance of the interaction with time $\left(\beta_{4}\right)$. Similarly, we present the difference in anxiety score for the parenting stress groups.

First we assessed the basic model as described for the anxiety scale and depression scale, then additionally we 
included the depression scale in the anxiety model, and the anxiety scale in the depression model. This second model eliminates overlap in associations between family variables and outcome due to common association with both depression and anxiety. Subsequently, we ran the basic model and added the five measures for parental mental health (the familial vulnerability indices for internalising and for externalising problems, and the three scales of the DASS). A strong attenuation of the coefficients for family variables would indicate that the effect of the family variables on anxiety is mostly the result of poor parental mental health. Similarly, we ran the basic model and added indicators of family composition (single-parent family and number of children in the family). We checked for interaction effects of the family variables with sex, socio-economic position and with parental mental health and family composition, to detect potential moderation of the association of family variables with anxiety by these other variables. None of these interactions was significant.

\section{Results}

Table 1 shows the baseline characteristics of the study population. Family dysfunction and parenting stress were correlated: $r=0.40(P<0.0001)$. Parental mental health problems were moderately correlated with family stress $(r=0.09-0.46)$ and weakly with adolescent anxiety scores at waves one and two $(r=0.05-0.10)$; these correlations were stronger for current parental internalising problems than for lifetime parental internalising problems (Table 2). The number of children in the family was not associated with family stress, and only marginally with anxiety scores at wave one. In single-parent households family stress was higher, but adolescent anxiety scores were not higher. The correlations are given in Table 2.

In Table 3 we present the results of the regression analyses. The table shows the findings adjusted for confounders only (first column), adjusted for confounders and depression (second column), and adjusted for confounders and parental mental health (third column). The difference in anxiety score between adolescents with families with dysfunction versus medium/high family functioning is $0.06(P=0.006)$ at age $10-12$ years and 0.009 $(P=0.62)$ at age $12-14$ years. The grey background indicates a significant interaction of family functioning with time $(P=0.02)$, indicating a stronger association at age 10-12 years, and a more favourable change in anxiety score over time in the family dysfunction group. At age 12-14 years family dysfunction was not associated with anxiety. Parenting stress was associated with current and future anxiety and the association was stable over time. All presented differences were small, given the average
Table 1 Baseline characteristics

\begin{tabular}{lll}
\hline & Mean (SD) & $N(\%)$ \\
\hline Male & - & $909(49)$ \\
Female & - & $966(51)$ \\
Age & $11.1(0.55)$ & - \\
Socio-economic position & & \\
Low & - & $415(22)$ \\
Middle & - & $952(51)$ \\
High & - & $508(27)$ \\
Number of children in family & & \\
One & - & $163(9)$ \\
Two & - & $935(50)$ \\
Three & - & $551(29)$ \\
Four or more & - & $226(12)$ \\
Single-parent household & & - \\
Parental mental health & & - \\
Lifetime internalising & $0.55(0.80)$ & - \\
Lifetime externalising & $0.13(0.41)$ & - \\
Current depression & $0.25(0.35)$ & - \\
Current anxiety & $0.16(0.29)$ & - \\
Current stress & $0.52(0.45)$ & - \\
Family variables & & \\
Family dysfunction & $1.77(0.36)$ & - \\
Parenting stress & $1.78(0.76)$ & \\
Anxiety score & $0.57(0.32)$ & \\
Depression score & $0.61(0.32)$ & \\
\hline & & \\
\hline
\end{tabular}

- indicates not applicable due to the type of variable

${ }^{\text {a }} N(\%)$ in family dysfunction group, or in high parenting stress group

scores for anxiety (e.g. total anxiety 0.57 at age 10 12 years).

Next we adjusted the anxiety model for depression and the depression model for anxiety. We expected the associations of parenting stress with anxiety to attenuate, whereas we expected less change in the associations of family dysfunction with anxiety, since the association with depression was stronger for parenting stress than for family dysfunction. This was confirmed in our analyses. After adjustment for depression, parenting stress was no longer associated with anxiety. After adjustment of the depression model for anxiety, the association with parenting stress remained significant. Thus, the association of parenting stress was stronger with depression than with anxiety. The cross-sectional association of family dysfunction with anxiety was less attenuated. Thus, most of the association of parenting stress with anxiety was not specific for anxiety, but was shared with depression, whereas the association of family dysfunction with anxiety was more specific for anxiety. 
Table 2 Associations of parental psychopathology and family composition with family dysfunction, parenting stress and anxiety scores

\begin{tabular}{|c|c|c|c|c|}
\hline & $\begin{array}{l}\text { Family } \\
\text { dysfunction }\end{array}$ & $\begin{array}{l}\text { Parenting } \\
\text { stress }\end{array}$ & $\begin{array}{l}\text { Anxiety } \\
10-12 \text { years }\end{array}$ & $\begin{array}{l}\text { Anxiety } \\
12-14 \text { years }\end{array}$ \\
\hline \multicolumn{5}{|l|}{ Parental mental health } \\
\hline Lifetime internalising & $0.13 * * *$ & $0.21 * * *$ & $0.07 *$ & 0.04 \\
\hline Lifetime externalising & $0.09 * * *$ & $0.12 * * *$ & 0.02 & -0.02 \\
\hline Current depression & $0.29 * * *$ & $0.39 * * *$ & $0.09 * * *$ & $0.05 *$ \\
\hline Current anxiety & $0.22 * * *$ & $0.29 * * *$ & $0.10^{* * *}$ & $0.07 * *$ \\
\hline Current stress & $0.28 * * *$ & $0.46^{* * *}$ & $0.10^{* * *}$ & $0.09 * *$ \\
\hline \multicolumn{5}{|l|}{ Family composition } \\
\hline Number of children & 0.03 & 0.02 & $0.05 *$ & 0.03 \\
\hline Number of parents & $0.15 * * *$ & $0.32 * * *$ & 0.01 & 0.01 \\
\hline
\end{tabular}

Values of parental mental health are Pearson correlations; values for number of children are Spearman correlations; and values of number of parents are the differences in scores for family dysfunction, parenting stress and anxiety scores and level of significance for $t$ test comparing single parent households with two-parent households

$* P<0.05 ; * * P<0.001 ; * * * P<0.0001$

Table 3 Difference in anxiety and depression scores (SE) between groups with and without family stress at age 10-12 years and age 1214 years

\begin{tabular}{|c|c|c|c|}
\hline & No adjustment & $\begin{array}{l}\text { Adjusted for } \\
\text { comorbidity }\end{array}$ & $\begin{array}{l}\text { Adjusted for } \\
\text { parental mental } \\
\text { health }\end{array}$ \\
\hline \multicolumn{4}{|l|}{ Anxiety } \\
\hline \multicolumn{4}{|l|}{$10-12$ years } \\
\hline Family dysfunction $^{\mathrm{a}}$ & $0.06(0.02)^{* *}$ & $0.03(0.02)^{*}$ & $0.05(0.02)^{*}$ \\
\hline Parenting stress ${ }^{\mathrm{a}}$ & $0.06(0.02) * * *$ & $0.003(0.01)$ & $0.05(0.02) * *$ \\
\hline \multicolumn{4}{|l|}{$12-14$ years } \\
\hline Family dysfunction $^{\mathrm{a}}$ & $0.009(0.02)$ & $-0.02(0.01)^{*}$ & $-0.001(0.02)$ \\
\hline Parenting stress ${ }^{\mathrm{a}}$ & $0.05(0.02)^{* *}$ & $0.007(0.01)$ & $0.03(0.02)^{*}$ \\
\hline \multicolumn{4}{|l|}{ Depression } \\
\hline \multicolumn{4}{|l|}{$10-12$ years } \\
\hline Family dysfunction $^{\mathrm{a}}$ & $0.04(0.02)$ & $-0.003(0.02)$ & $0.02(0.02)$ \\
\hline Parenting stress ${ }^{\mathrm{a}}$ & $0.09(0.02)^{* * *}$ & $0.05(0.01)^{* * *}$ & $\mathbf{0 . 0 7}(\mathbf{0 . 0 2})^{* * *}$ \\
\hline \multicolumn{4}{|l|}{$12-14$ years } \\
\hline Family dysfunction $^{\mathrm{a}}$ & $0.06(0.02)^{* *}$ & $0.05(0.02)^{* * *}$ & $0.04(0.02)^{*}$ \\
\hline Parenting stress ${ }^{\mathrm{a}}$ & $0.06(0.02) * * *$ & $0.03(0.01)^{*}$ & $0.04(0.02)^{*}$ \\
\hline
\end{tabular}

All models were adjusted for socio-economic position and sex. In bold are significant findings $(P<0.05)$. In italics and in bold italics, the change in time of the anxiety/depression score differs $(P<0.05)$ by the value of the family variable, and thus the difference in anxiety score by family functioning or by parenting stress is not the same at age 10-12 years and age 10-14 years

$* P<0.05 ; * * P<0.01$; *** $P<0.001$

${ }^{a}$ Dysfunction versus medium/high family functioning and high versus medium/low parenting stress

In the following two models we adjusted for parental mental health and for family composition. A strong attenuation of the associations of family dysfunction and parenting stress with anxiety would indicate that the effect of family variables on anxiety is mostly the result of parental mental health, or family composition. Adjustment for parental mental health attenuated the differences in Table 3 a little (third column). Adjustment for family composition did not affect the findings (data not shown).

\section{Discussion}

This study examined the role of family dysfunction and parenting stress (two indicators of family stress) in anxiety in adolescents. The findings showed that family dysfunction and high parenting stress were associated with anxiety. Family dysfunction was not associated with future anxiety, whereas high parenting stress was. Furthermore, family dysfunction was more strongly associated with anxiety than 
with depression, whereas parenting stress was more strongly associated with depression. Parental mental health problems explained part of the association of family dysfunction and parenting stress with anxiety. Family composition did not explain these associations. The associations found in the study were weak. Taking into account that this study involved a normal-population sample of adolescents, in which we expect relatively low levels of family stress and of anxiety and depression symptoms as compared to, for example high-risk samples and clinical samples, and the multifactorial aetiology of anxiety, we feel that higher effect sizes could not be expected.

The association of family dysfunction with anxiety was not stable from age 10-12 to 12-14 years: family dysfunction was associated with concurrent anxiety (age 1012 years) only. This may indicate that (the perception of) family dysfunction by parents is provoked by anxiety of the child. Alternatively, family dysfunction may indeed be a risk factor for anxiety, but only in early adolescence and no longer in middle and late adolescence. As adolescents grow up it would be expected that other relationships, such as with peers, develop and influence the adolescent's well-being and coping resources [3]. These changes may be particularly triggered by events such as the transition from primary to secondary school. The 2-year follow-up period of our study included this transition. Therefore, family dysfunction may be a more prominent risk factor in younger than in older adolescents. Indeed, in the few other prospective community studies also no associations were found with depression or anxiety in adolescents older than 12 years [22, 38, 41]. We did not find any prospective community studies on family dysfunction and anxiety in younger adolescents. Thirdly, family functioning may have been unstable over the follow-up period, so that families that had higher dysfunction at age 10-12 years might not have dysfunction at age 12-14 years or during the follow-up.

High parenting stress was associated with both current and future anxiety. The associations of parenting stress thus did not decrease with age. Similar (small) effects have also been found for both anxiety and depression in the few other prospective studies in community samples, in younger children [2, 27], and in offspring of parents without (history of) major depression [31]. The association of parenting stress was strongest for depression. Though few studies are available on the specific effects of family variables on anxiety and depression, findings indicate that specific parenting domains (e.g. rejection) are stronger associated with depression than with anxiety [44].

After adjustment for parental mental health problems, the associations of family stress attenuated slightly (with approximately $10 \%$ ), which indicates that part of the family stress associations may be the result of parental mental health problems. This has been shown before for the effect of parenting styles [21]. Alternatively, a persistent question in parenting stress research has been whether measures of parenting stress are distinctly different from measures of parental psychopathology $[8,12]$. In this community sample, higher parenting stress was still associated with adolescent anxiety and depression after adjustment for measures of lifetime and current parental mental health problems, indicating that parenting stress is an independent construct. Parenting stress is a normal consequence of being a parent, regardless of parents' own and their children's attributes, their socio-economic position, and their support networks [12]. This community study showed that also moderate to high levels of parenting stress are associated with internalising problems in adolescents.

This study has several strengths including the prospective design, the large representative community sample, and different informants for family stress (parent) and anxiety symptoms (adolescent). Some limitations of our study should also be mentioned: (1) Because family stress was measured at baseline only, we could not study the effect of changes in family stress on adolescent anxiety, the effect of adolescent anxiety on family stress, or the relative importance of family stress as predictor and as a consequence of anxiety symptoms. Other studies have demonstrated both directions of the effect [35], and in future prospective studies it will be worth measuring family stress repeatedly. As in every prospective study, this study was at risk for selective attrition. (2) Selective attrition was found only for number of parents in the household, parental socio-economic position, and parental externalising problems. Neither of these variables was strongly associated with both family stress variables and anxiety and depression scale; therefore, bias due to selective attrition is unlikely. (3) Another limitation is that parental mental health was not assessed with a diagnostic interview. (4) We did not have information about marital quality. Tension between parents may be a third source of family stress, not covered by family dysfunction or parenting stress. (5) For testing mediation, ideally at least three waves of assessment are required. The longitudinal nature of TRAILS will allow us to investigate mediation models better in the future. (6) The findings can probably be generalised to other community samples, though not necessarily to clinical or high-risk samples. The effects found in this community sample were small, which may partly be attributed to the generally good health of this sample, and the relatively low levels of family stress [13]. In a clinical or high-risk sample both levels of anxiety and of family stress may be higher and family stress may be a stronger predictor of anxiety.

In conclusion, family dysfunction was associated with current anxiety, but not associated with future anxiety. Parenting stress was associated with anxiety and depression both concurrently and in the future. Parenting stress was 
more a risk factor for symptoms of depression, than for symptoms of anxiety. The associations were modest and the understanding of the origins of adolescents' anxiety will require identifying other factors than family stress that account for more of the variance. However, combining the findings with findings that parenting stress was related to a less favourable treatment outcome in children with anxiety problems [9], suggests that information about parenting stress in children with subclinical anxiety problems may be used to predict risk of developing persistent symptoms, and to optimize treatment. Furthermore, for parents it is important to know that the parenting stress they experience is a risk factor for the development of internalising problems in their children. This may encourage them to seek help in overcoming the problems underlying their parenting stress.

Acknowledgments This research is part of the Tracking Adolescents' Individual Lives Survey (TRAILS), a collaboration of research centers from the University of Groningen, Erasmus Medical Center of Rotterdam, University of Nijmegen, University of Utrecht, and Trimbos Institute the Netherlands. TRAILS has been financially supported by grants from the Netherlands Organization for Scientific Research (GB-MW 940-38-011, GB-MAGW 480-01-006, GBMAGW 457-03-018, NWO 175.010.2003.005, ZonMw 100-001-001, ZonMw 60-60600-98-018), the Dutch Ministry of Justice (WODC), the Sophia Foundation for Medical Research, and the participating research centers. F. van Oort was financially supported by the Sophia Foundation for Medical Research (EC-SAR). We thank all children, their parents/caregivers and their teachers who willingly participated and everyone who worked on this project and made it possible.

Open Access This article is distributed under the terms of the Creative Commons Attribution Noncommercial License which permits any noncommercial use, distribution, and reproduction in any medium, provided the original author(s) and source are credited.

\section{References}

1. Abidin RR (1983) Parenting stress and the utilization of pediatric services. Child Health Care 11:70-73

2. Abidin RR, Jenkins CL, Mcgaughey MC (1992) The relationship of early family variables to children's subsequent behavioraladjustment. J Clin Child Psychol 21:60-69

3. Arnett JJ (2000) Emerging adulthood. A theory of development from the late teens through the twenties. Am Psychol 55:469-480

4. Bijl RV, Ravelli A, van Zessen G (1998) Prevalence of psychiatric disorder in the general population: results of the Netherlands Mental Health Survey and Incidence Study (NEMESIS). Soc Psychiatry Psychiatr Epidemiol 33:587-595

5. Bogels SM, Brechman-Toussaint ML (2006) Family issues in child anxiety: attachment, family functioning, parental rearing and beliefs. Clin Psychol Rev 26:834-856

6. Byles J, Byrne C, Boyle MH, Offord DR (1988) Ontario Child Health Study: reliability and validity of the general functioning subscale of the McMaster Family Assessment Device. Fam Process 27:97-104

7. Chorpita BF, Yim L, Moffitt C, Umemoto LA, Francis SE (2000) Assessment of symptoms of DSM-IV anxiety and depression in children: a revised child anxiety and depression scale. Behav Res Ther 38:835-855

8. Costa NM, Weems CF, Pellerin K, Dalton R (2006) Parenting stress and childhood psychopathology: an examination of specificity to internalizing and externalizing symptoms. J Psychopathol Behav Assess 28:113-122

9. Crawford AM, Manassis K (2001) Familial predictors of treatment outcome in childhood anxiety disorders. J Am Acad Child Adolesc Psychiatry 40:1182-1189

10. De Brock AJLL, Gerris JRM, Vermulst AA (1992) NOSI, Nijmeegse Ouderlijke Stress Index. Swets en Zeitlinger, Lisse

11. de Winter AF, Oldehinkel AJ, Veenstra R, Brunnekreef JA, Verhulst FC, Ormel J (2005) Evaluation of non-response bias in mental health determinants and outcomes in a large sample of pre-adolescents. Eur J Epidemiol 20:173-181

12. Deater-Deckard K (1998) Parenting stress and child adjustment: some old hypotheses and new questions. Clin Psychol Sci Pract $5: 314-332$

13. Deater-Deckard K, Scarr S (1996) Parenting stress among dualearner mothers and fathers: are there gender differences? J Fam Psychol 10:45-59

14. Dickstein S, Seifer R, Hayden LC, Schiller M, Sameroff AJ, Keitner G, Miller I, Rasmussen S, Matzko M, Magee KD (1998) Levels of family assessment: II. impact of maternal psychopathology on family functioning. J Fam Psychol 12:23-40

15. Epstein NB, Baldwin L, Bishop D (1983) The McMaster Family Assessment Device. J Marital Fam Ther 9:171-180

16. Ferdinand RF, van Lang ND, Ormel J, Verhulst FC (2006) No distinctions between different types of anxiety symptoms in preadolescents from the general population. J Anxiety Disord 20:207-221

17. Grant KE, Compas BE, Stuhlmacher AF, Thurm AE, McMahon SD, Halpert JA (2003) Stressors and child and adolescent psychopathology: moving from markers to mechanisms of risk. Psychol Bull 129:447-466

18. Henry JD, Crawford JR (2005) The short-form version of the Depression Anxiety Stress Scales (DASS-21): construct validity and normative data in a large non-clinical sample. Br J Clin Psychol 44:227-239

19. Hudson JL, Rapee RM (2001) Parent-child interactions and anxiety disorders: an observational study. Behav Res Ther 39:1411-1427

20. Kendler KS, Prescott CA, Myers J, Neale MC (2003) The structure of genetic and environmental risk factors for common psychiatric and substance use disorders in men and women. Arch Gen Psychiatry 60:929-937

21. Leinonen JA, Solantaus TS, Punamaki R-L (2003) Parental mental health and children's adjustment: the quality of marital interaction and parenting as mediating factors. J Child Psychol Psychiatry 44:227-241

22. Lieb R, Wittchen HU, Hofler M, Fuetsch M, Stein MB, Merikangas KR (2000) Parental psychopathology, parenting styles, and the risk of social phobia in offspring: a prospectivelongitudinal community study. Arch Gen Psychiatry 57:859866

23. Lindhout I, Markus M, Hoogendijk T, Borst S, Maingay R, Spinhoven P, van Dyck R, Boer F (2006) Childrearing style of anxiety-disordered parents. Child Psychiatry Hum Dev 37:89102

24. Lovibond PF, Lovibond SH (1995) The structure of negative emotional states: comparison of the Depression Anxiety Stress Scales (DASS) with the Beck Depression and Anxiety Inventories. Behav Res Ther 33:335-343

25. Manassis K, Bradley S, Goldberg S, Hood J, Swinson RP (1994) Attachment in mothers with anxiety disorders and their children. J Am Acad Child Adolesc Psychiatry 33:1106-1113 
26. Manassis K, Hood J (1998) Individual and familial predictors of impairment in childhood anxiety disorders. J Am Acad Child Adolesc Psychiatry 37:428-434

27. McCarty CA, Zimmerman FJ, Digiuseppe DL, Christakis DA (2005) Parental emotional support and subsequent internalizing and externalizing problems among children. J Dev Behav Pediatr 26:267-275

28. McLeod BD, Wood JJ, Weisz JR (2007) Examining the association between parenting and childhood anxiety: a meta-analysis. Clin Psychol Rev 27:155-172

29. Messer SC, Beidel DC (1994) Psychosocial correlates of childhood anxiety disorders. J Am Acad Child Adolesc Psychiatry 33:975-983

30. Miller IW, Epstein N, Bishop D, Keitner G (1985) The McMaster Family Assessment Device: reliabilty and validity. J Marital Fam Ther 11:345-356

31. Nomura Y, Wickramaratne PJ, Warner V, Mufson L, Weissman MM (2002) Family discord, parental depression, and psychopathology in offspring: ten-year follow-up. J Am Acad Child Adolesc Psychiatry 41:402-409

32. Onatsu-Arvilommi T, Nurmi JE, Aunola K (1998) Mothers' and fathers' well-being, parenting styles, and their children's cognitive and behavioural strategies at primary school. Eur J Psychol Educ 13:543-556

33. Ormel J, Oldehinkel AJ, Ferdinand RF, Hartman CA, De Winter AF, Veenstra R, Vollebergh W, Minderaa RB, Buitelaar JK, Verhulst FC (2005) Internalizing and externalizing problems in adolescence: general and dimension-specific effects of familial loadings and preadolescent temperament traits. Psychol Med $35: 1825-1835$

34. Rapee RM (1997) Potential role of childrearing practices in the development of anxiety and depression. Clin Psychol Rev 17:4767

35. Reitz E, Dekovic M, Meijer AM (2006) Relations between parenting and externalizing and internalizing problem behaviour in early adolescence: child behaviour as moderator and predictor. $\mathbf{J}$ Adolesc 29:419-436

36. Ritchie KL, Holden GW (1998) Parenting stress in low income battered and community women: effects on parenting behavior. Early Educ Dev 9:97-112

37. Siqueland L, Kendall PC, Steinberg L (1996) Anxiety in children: perceived family environments and observed family interaction. J Clin Child Psychol 25:225-237

38. Sourander A, Aromaa M, Pihlakoski L, Haavisto A, Rautava P, Helenius H, Sillanpaa M (2006) Early predictors of deliberate self-harm among adolescents. A prospective follow-up study from age 3 to age 15. J Affect Disord 93:87-96

39. Spence SH (1997) Structure of anxiety symptoms among children: a confirmatory factor-analytic study. J Abnorm Psychol 106:280-297

40. Storksen I, Roysamb E, Holmen TL, Tambs K (2006) Adolescent adjustment and well-being: effects of parental divorce and distress. Scand J Psychol 47:75-84

41. Tamplin A, Goodyer IM (2001) Family functioning in adolescents at high and low risk for major depressive disorder. Eur Child Adolesc Psychiatry 10:170-179

42. Twisk JWR (2003) Applied longitudinal data analysis for epidemiology: a practical guide. Cambridge University Press, Cambridge

43. Veenstra R, Lindenberg S, Oldehinkel AJ, De Winter AF, Verhulst FC, Ormel J (2005) Bullying and victimization in elementary schools: a comparison of bullies, victims, bully/victims, and uninvolved preadolescents. Dev Psychol 41:672-682

44. Vulic-Prtoric A, Macuka I (2006) Family and coping factors in the differentiation of childhood anxiety and depression. Psychol Psychother 79:199-214

45. Wood JJ, McLeod BD, Sigman M, Hwang WC, Chu BC (2003) Parenting and childhood anxiety: theory, empirical findings, and future directions. J Child Psychol Psychiatry 44:134-151 